\title{
Naissance Anormale Du Tronc Coronaire Gauche A Partir De L'artere Pulmonaire : à propos d'un cas (Anomalous Left Coronary Artery From Pulmonary Artery: about a case)
}

\section{Ravaoavy Hariniaina ${ }^{\star, \dagger, 1}$, Rakotojoelimaria Haganiaina Elsa ${ }^{2}$, Ramiandrisoa Lahatriniavo Ritchy ${ }^{3}$, Miandrisoa Rija Mikhaël ${ }^{4}$, Ralamboson Solofo Andrianarivelo, Rakotoarimanana Solofo ${ }^{6}$}

\footnotetext{
${ }^{1}$ Service des Maladies Cardiovasculaires, Centre Hospitalier de Soavinandriana, Antananarivo, Madagascar.

${ }^{2}$ Centre Hospitalier Universitaire Mère Enfant Tsaralalana, Antananarivo, Madagascar

${ }^{3}$ Service Polyclinique Médicale, Centre Hospitalier Universitaire Andohatapenaka, Antananarivo, Madagascar.

${ }_{4}^{4}$ Service des Maladies Cardiovasculaires, Centre Hospitalier de Soavinandriana, Antananarivo, Madagascar.

${ }^{5}$ Service des Maladies Cardiovasculaires, Centre Hospitalier de Soavinandriana, Antananarivo, Madagascar.

${ }^{6}$ Service des Soins Intensifs Cardiologiques, CHU Befelatanana, Antananarivo, Madagascar.
}

(1) DOI: https://doi.org/10.15520/jcmro.v2i10.224

Accepted 23-10-2019; Received 01-10-2019; Publish Online 24-10-2019

Reviewed By:

Dr. S. Niknamian

Department:

Reviewer/CMRO

\begin{abstract}
Anomalous left coronary artery from pulmonary artery ALCAPA, is a rare congenital heart disease. It affects one child in 300,000 births. It causes myocardial ischemia leading to impaired left ventricle systolic function and signs of left heart failure. Several authors have already reported cases of ALCAPA. We are reporting for the first time a case seen in Madagascar. This is a Franco-Malagasy child aged 4 months and 3 weeks, hospitalized at the Mother and Child University Hospital of Tsaralalana for progressively worsening dyspnea. At entry, the infant presented with cyanosis and signs of cardiogenic shock: hypotension, oligoanuria and cold extremities. The physical examination essentially found pulmonary rales, a $2 / 6$ systolic murmur of mitral insufficiency and hepatomegaly. Chest X-ray showed significant cardiomegaly. The electrocardiogram found a $\mathrm{Q}$ wave of necrosis in DI, AVL and V2 to V6. The echocardiogram coupled with Doppler performed at the Soavinandriana Hospital concluded that the left coronary artery was connected to the pulmonary artery. The progression after 6 days in hospital was marked by the disappearance of signs of shock and dyspnea after treatment with Dobutamine, diuretics and Salbutamol. The child was proposed for a surgical cure, the gold standard of treatment for ALCAPA.
\end{abstract}

Resume:

La naissance anormale du tronc coronaire gauche à partir de l'artère pulmonaire ou ALCAPA, de l'anglais anomalous left coronary artery from pulmonary artery ou encore syndrome de Bland-White-Garland, est une pathologie congénitale rare. Elle affecte un enfant sur 300000 naissances. Elle entraine une ischémie myocardique à l'origine d'une altération de la fonction systolique du ventricule gauche et des signes d'insuffisance cardiaque gauche. Plusieurs auteurs ont déjà rapporté les cas de ALCAPA. Nous rapportons pour la première fois un cas vu à Madagascar. Il s'agit d'un nourrisson Franco-Malgache de 4 mois et 3 semaines, hospitalisé au Centre Hospitalier Universitaire Mère-Enfant de Tsaralalana pour une dyspnée d'aggravation progressive. A l'entrée, le nourrisson présentait une altération de l'état général, cyanose et des signes de choc cardiogénique : hypotension artérielle, oligoanurie et extrémités froides. L'examen physique retrouvait essentiellement des râles crépitants diffus, un souffle systolique $2 / 6$ d'insuffisance mitrale et une hépatomégalie. La radiographie du thorax de face montrait une cardiomégalie importante. L'électrocardiogramme a retrouvé une onde $\mathrm{Q}$ de nécrose en DI, AVL et de V2 jusqu'en V6. L'échocardiogramme couplé au Doppler réalisé au Centre Hospitalier de Soavinandriana a conclu à l'anomalie de connexion du tronc de l'artère coronaire gauche à partir de l'artère pulmonaire. L'évolution après 6 jours d'hospitalisation a été marquée par la disparition des signes de choc et de la dyspnée après un traitement par Dobutamine, diurétiques et Salbutamol. L'enfant a été proposé pour une cure chirurgicale, le gold standard du traitement de l'ALCAPA. 


\section{INTRODUCTION:}

La naissance anormale du tronc de la coronaire gauche à partir de l'artère pulmonaire ou encore le syndrome de Bland-White-Garland est une pathologie congénitale rare [1]. Pendant la période néonatale, comme la pression dans l'artère pulmonaire est basse, le flux de l'artère coronaire gauche va se jeter dans l'artère pulmonaire. Ce phénomène est à l'origine d'un shunt gauche-droit et du vol coronarien qui vont entrainer une atteinte myocardique par diminution de sa perfusion. L'insuffisance cardiaque gauche, puis globale, va par la suite s'installer. Non traitée, cette pathologie entraîne une mortalité de $90 \%$ dans la première année de vie par une ischémie myocardique et une insuffisance cardiaque [2] . Le traitement définitif est la chirurgie qui consiste à aboucher le tronc de l'artère coronaire gauche au niveau de l'aorte. Nous rapportons le cas d'un nourrisson de 4 mois et 3 semaines, présentant un syndrome d'insuffisance cardiaque globale avec cyanose et altération de l'état général. L'électrocardiogramme et l'échocardiographie couplé au Doppler ont permis de poser le diagnostic.

\section{OBSERVATION:}

Il s'agit un petit garçon Franco-Malgache de 4 mois et 3 semaines, originaire de Nosy Be. Il a été hospitalisé au mois de septembre 2019 au Centre Hospitalo-Universitaire MèreEnfant de Tsaralalana pour une difficulté respiratoire. Son histoire se résume à une toux sèche à répétition, non dyspnéisante mais associée à des sifflements, évoluant depuis l'âge de 3 mois. Il a eu un épisode de virose respiratoire une semaine avant son admission. L'aggravation des signes respiratoires et la réapparition de la toux, qui est devenue dyspnéisante et cyanosante, a motivé le transfert dans notre service.

Dans ses antécédents, c'est un enfant unique, issu d'une grossesse bien suivie par un médecin généraliste de ville, bien adapté en néonatale à la suite d'un accouchement par voie basse, avec un poids de naissance de 3500 grammes. Et du côté familiale, on rapporte une atopie chez le père.

L'examen clinique à l'entrée retrouvait un bébé en mauvais état général, obnubilé, avec un état hémodynamique instable. Il présentait des signes de luttes respiratoires, une cyanose labiales et des extrémités ainsi que des signes de choc cardiogénique : une oligoanurie, froideur des extrémités et marbrure. Son poids était à $5,880 \mathrm{~kg}$ et la taille $64 \mathrm{~cm}$. La pression artérielle était de $60 / 30 \mathrm{mmHg}$ et la fréquence cardiaque à 190 battements par minute. La saturation en oxygène était à $80 \%$ à l'air ambiant et la température à $37^{\circ} \mathrm{C}$. L'auscultation cardiaque retrouvait des bruits du cœur normaux, un souffle systolique $2 / 6$ d'insuffisance mitrale, unB2 pulmonaire normal. L'auscultation pulmonaire révélait des râles crépitants diffus mais asymétriques sans

\footnotetext{
* Corresponding author.

† Email: ravaoavy@gmail.com
}

syndrome d'épanchement pleural. On notait également une hépatomégalie avec une flèche hépatique mesurée à $8 \mathrm{~cm}$ et un reflux hépato-jugulaire.

La radiographie du thorax de face objectivait une cardiomégalie importante avec un rapport cardio-thoracique à 0.65 Figure 1.

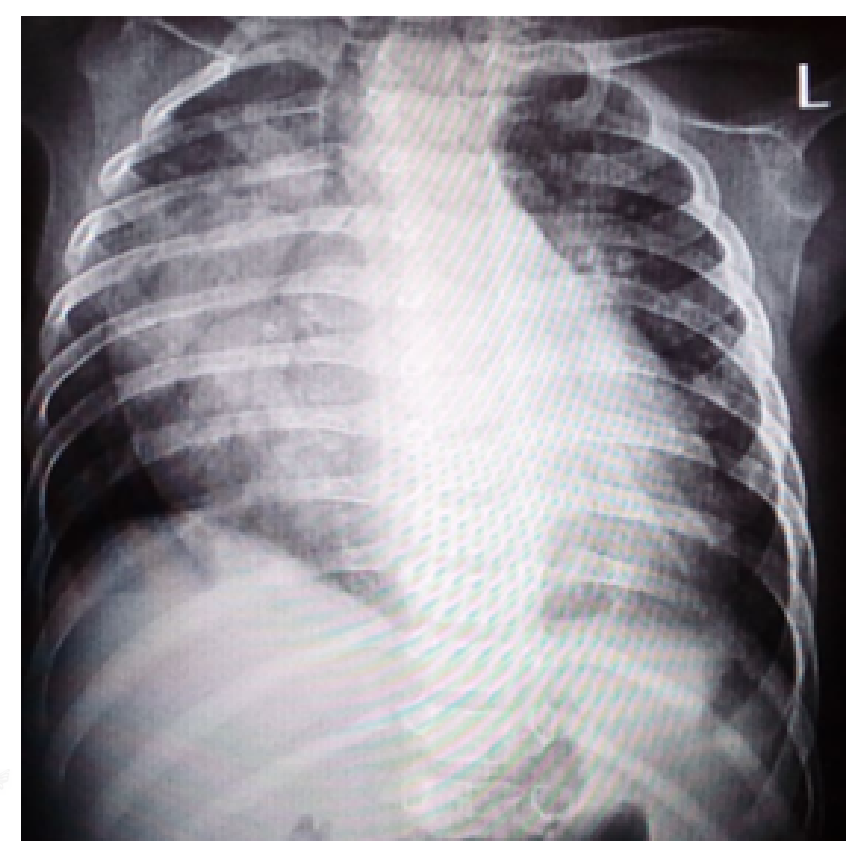

Figure 1. radiographie thoraciquemontrant la cardiomégalie radiologique

L'électrocardiogramme (ECG) de surface inscrivait une tachycardie sinusale régulière à 150 cycles par minute, une onde Q profonde et large en DI, AVL et de V2 jusqu'en V6 Figure 2 .

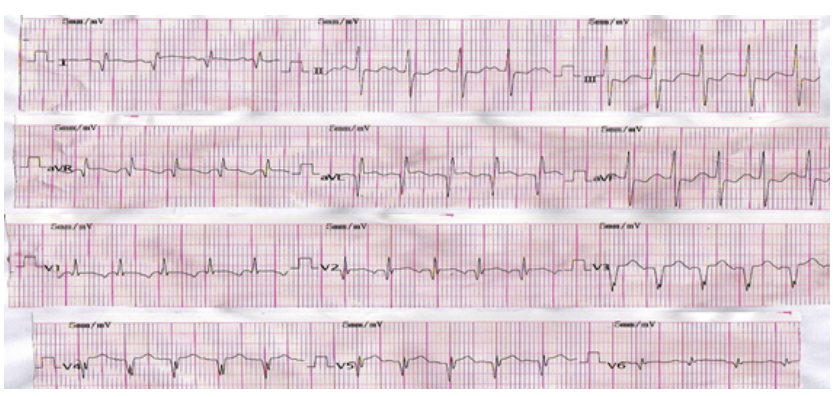

Figure 2. ECG de surface montrantune tachycardie sinusale, une onde Q large et profonde en DI, AVL et de V2jusqu'en V6

L'échocardiographie-Doppler réalisé au Centre Hospitalier de Soavinandriana retrouvait une dilatation importante des cavités gauches avec un ventricule gauche $(\mathrm{VG})$ à $+6,53$ z score Figure 3, un trouble de la cinétique segmentaire du VG à type d'akinésie antéro-septo-médian, antéro-apicale ; une altération de la fonction systolique du VG avec une fraction d'éjection du VG à $27 \%$ en Simpson Biplan. Le pilier antéro-latéral de la mitrale était hyperéchogène, on 
note une akinésie du segment au niveau de l'insertion pariétale de ce pilier. Il y avait une fuite mitrale modérée avec une valve mitrale fine et souple, sans anomalie congénitale. Le jet de cette fuite mitrale était excentré (Figure 4), il s'agissait d'une insuffisance mitrale secondaire d'origine ischémique. L'artère coronaire gauche était bien visible et ne se connectait pas dans l'aorte (Figure 5), l'artère coronaire droite était bien en place. L'examen avait conclu à une anomalie de connexion du tronc coronaire gauche à partir de l'artère pulmonaire avec aspect d'une cardiomyopathie ischémique et altération sévère de la fonction systolique du VG.

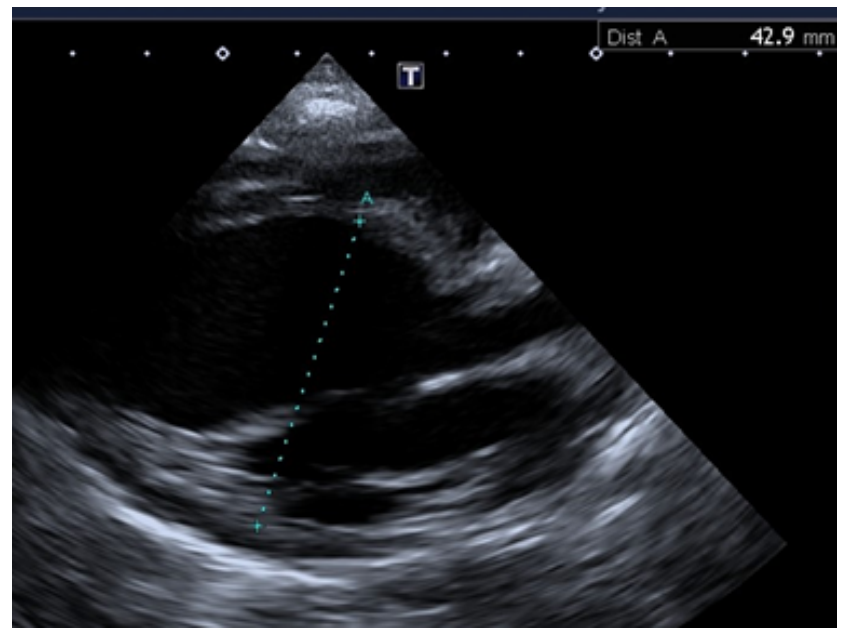

Figure 3. image échocardiographiqueen 2D montrant le VG di-laté

Durant l'hospitalisation, l'enfant avait reçu comme traitement un tonicardiaque (Dobutamine à $5 \gamma / \mathrm{kg} / \mathrm{min}$ à l'entrée puis sevré), des diurétiques (Furosémide $1 \mathrm{mg} / \mathrm{kg}$ et Spironolactone $1 \mathrm{mg} / \mathrm{kg}$ toutes les 6 heures), Salbutamol (en aérosol toutes les 4 heures puis 6 heures et en spray pendant une semaine). L'alimentation était fractionné par l'intermédiaire d'une sonde naso-gastrique. L'évolution clinique était bonne après les 6 jours d'hospitalisation avec amélioration de l'état général, disparition de la dyspnée et de la cyanose. Il restait une petite hépatomégalie. Nous avons poursuivi le traitement diurétique et nous l'avons adressé en chirurgie, le gold standard du traitement de l'ALCAPA.

\section{COMMENTAIRE:}

La naissance anormale du tronc coronaire gauche à partir de l'artère pulmonaire ou communément appelé « anomalous left coronary artery from pulmonary artery » (ALCAPA) est une connexion anormale de l'artère coronaire gauche dans l'artère pulmonaire. Elle est aussi connue sous le nom de syndrome de Bland-White-Garland. Elle a été décrite pour la première fois par ces derniers en 1933 [1] bien qu'en 1866 et 1908 on en a entendu parler brièvement $[3,4]$. C'est une anomalie congénitale rare affectant

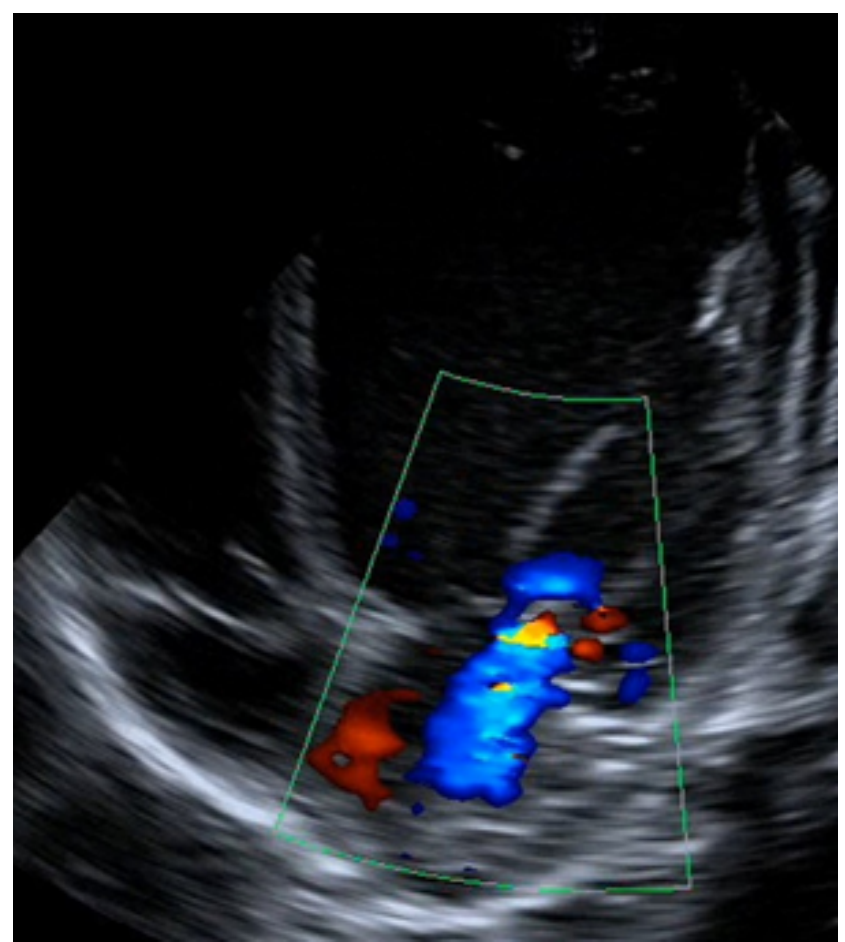

Figure 4. imageéchocardiographique couplé au Doppler montrant le jet excentré de la fuitemitrale

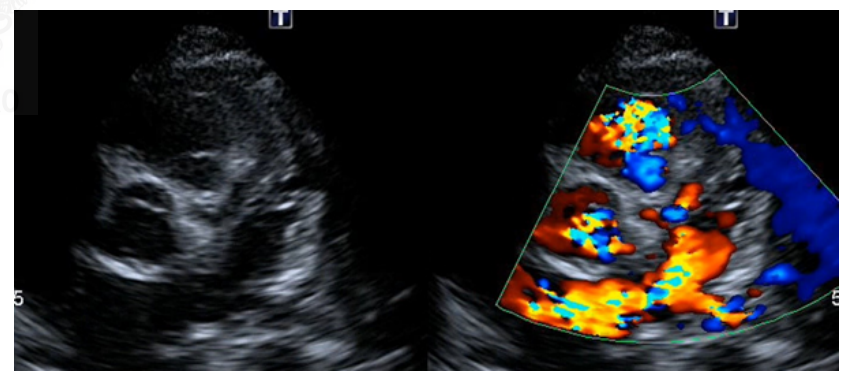

Figure 5. imageéchocardiographique couplé au Dopplermontrant le non abouchement du tronc coronaire gauche dans l'aorte

un enfant sur 300000 naissances [5]. Sur le plan physiopathologique, c'est l'artère coronaire droite qui assure de manière prépondérante la perfusion myocardique. La coronaire gauche naissant de l'artère pulmonaire va vasculariser le myocarde ventriculaire gauche par du sang pauvre en oxygène susceptible d'induire des lésions d'ischémie à l'effort ou bien elle est perfusée de façon rétrograde à partir de la coronaire droite et se vide dans l'artère pulmonaire lorsque les pressions pulmonaires baissent, entrainant un vol coronaire [6].

Plusieurs auteurs ont déjà rapporté des cas de cette pathologie, notamment des cas similaires au notre [7,8]. La particularité de notre cas est que c'est le seul et le premier à être rapporté à Madagascar. Il s'agit d'un nourrisson de 4 mois et trois semaines hospitalisé dans l'hôpital mère enfant de Tsaralalana pour un syndrome d'insuffisance cardiaque globale sévère avec hypoxie et cyanose. L'ALCAPA peut se manifester, soit à très bas âge, soit plus tardi- 
vement et de façons beaucoup plus modérée [9]. La manifestation à très bas âge se situe vers le $2^{\text {ème }}$ mois de vie. Elle est asymptomatique in utero et dans les premières heures de vie. Plus tard à cause de la diminution post-natale des pressions pulmonaires, un flux rétrograde est engendré au sein de l'artère coronaire gauche et favorise l'ischémie myocardique [10].L'enfant présente alors des signes de décompensation cardiaque surtout gauche tels les signes de lutte respiratoire et polypnée avec des congestions pulmonaires comme la présentation clinique de notre cas. La forme plus tardive ne se manifeste qu'à l'adolescence voire à l'âge adulte du fait de réseau collatéral relativement suffisant entre les coronaires. On assiste alors à un angor à l'effort, à l'apparition de souffle, mais parfois aussi à une mort subite [10]. Sur le plan paraclinique, une cardiomégalie radiologique importante est souvent rapportée comme chez notre cas. Une onde $Q$ large et profonde en D1 et AVL à l'ECG est très évocatrice et typique bien que Hoffman J.I. a rapporté des ECG dans la limite de la normale chez quelques patients atteint d'ALCAPA [11]. L'échocardiographie est un examen clé chez l'enfant mais difficile à réaliser chez l'adulte. Ce n'est pas toujours possible chez l'adulte de trouver les origines des artères coronaires. Chez notre patient, il a été aisé de trouver le non-abouchement du tronc de l'artère coronaire gauche dans l'aorte. Dans d'autres cas, on peut voir le flux rétrograde dans l'artère pulmonaire à partir de l'artère coronaire gauche [12]. Ce qui n'a pas été observé dans le nôtre. D'autres signes échocardiographiques, à rechercher dans le cas de l'ALCAPA, étaient présents chez notre patient tels que la dysfonction systolique du VG, le trouble de la cinétique segmentaire du VG, l'aspect hyperéchogène du pilier de la valve mitrale et surtout la fuite mitrale excentrée d'origine ischémique qui a une valeur diagnostique importante voire pathognomonique dans l'ALCAPA [9]. Les autres examens paracliniques tels que l'Imagerie par Résonnance Magnétique cardiaque, le scanner multibarrette ou le coroscanner ainsi que l'aortographie n'ont pas été réalisés devant la non disponibilité locale de ces plateaux techniques.

L'évolution clinique était bonne sous diurétiques et l'enfant est sorti au bout de 10 jours d'hospitalisation. Il a été par la suite adressé en chirurgie, en évacuation sanitaire, pour le traitement chirurgicale de sa pathologie.

\section{CONCLUSION:}

L'ALCAPA est une anomalie congénitale rare. Les signes d'insuffisance cardiaque gauche et l'onde $Q$ de nécrose en DI et AVL chez le nourrisson font évoquer le diagnostic. L'échocardiographie-Doppler le confirme et recherche ses complications qui sont essentiellement la dilatation et la dysfonction systolique du VG, la fuite mitrale secondaire. Ces examens qui sont aisément réalisables à Madagascar doivent permettre de poser le diagnostic d'ALCAPA afin d'optimiser le traitement qui est chirurgical.

Conflits d'intérêts: Les auteurs ne déclarent aucun conflit d'intérêt.
Contributions des auteurs: Tous les auteurs ont contribué à la conduite de ce travail. Tous les auteurs déclarent également avoir lu et approuvé la version finale du manuscrit.

\section{REFERENCES}

[1] Bland EF, White PD, Garland J. Congenital anomaly of coronary artery : report of an unusual case in association with cardiac hypertrophy. Am Heart J. 1933;8:787-801.

[2] Wollenek G, Domanig E, Salzer-Muhar U. Anomalous origin of the left coronary artery: a review of surgical management in 13 patients. J Cardiovasc Surg. 1993;34:399-405.

[3] Two cases of an abnormal coronary artery of the heart arising from the pulmonary artery: with some remarks upon the effect of this anomaly in producing cirsoid dilatation of the vessels. J Anat Physiol. 1885;20:26-29.

[4] Wilson CL, Dlabal PW, Holeyfield RW. Anomalous origin of left coronary artery from pulmonary artery: Case report and review of literature concerning teen-agers and adults. J Thorac Cardiovasc Surg. 1977;73:887-893.

[5] Erdinc M, Hosgor K, Karahan O. Repair of anomalous origin of the left coronary artery arising from right pulmonary artery with rolled-conduit-extended reimplantation in an adult. J Card Surg. 2011;26:604-607.

[6] Weigand J, Marshall D, Bacha C, EA. Repair of anomalous left coronary artery from pulmonary artery in the Modern Era : Preoperative Predictors of Immediate Postoperative Outcomes and Long Term Cardiac Followup. Pediatr Cardiol. 2015;36:489-497.

[7] Sahnoun I, Abid L, Abid D, Triki F. La naissance anormale $\mathrm{du}$ tronc coronaire gauche à partir de l'artère pulmonaire : étiologie de défaillance cardiaque chez le nourrisson. JI M Sfax. 2008;15:23-25.

[8] Gribaa M, Slim HB, Salem. Anomalous origin of the left coronary artery from the pulmonary artery presenting as dilated cardiomyopathy: a case report. Journal of Medical Case Reports. 2014;8:170-170.

[9] Bilderling CGD, Geurten J, Evrard. Quand l'insuffisance cardiaque se cache derrière la bronchiolite. Louvain Med. 2017;136(4):245-249.

[10] Pena E, Nguyen ET, Merchant N. ALCAPA Syndrome: Not Just a Pediatric Disease. Radiographics. 2009;29:553-565.

[11] Hoffman JI. Electrocardiogram of anomalous of left coronary artery from the pulmonary artery in infants. Pediatric Cardiol. 2013;34:489-491.

[12] Sanjay T, Apurba KS, Kurur SV. Lessons from a case of anomalous the left coronary artery fromhe pulmonary artery. Ind J Thorac Cardiovasc Surg. 2005;21:171-172. 